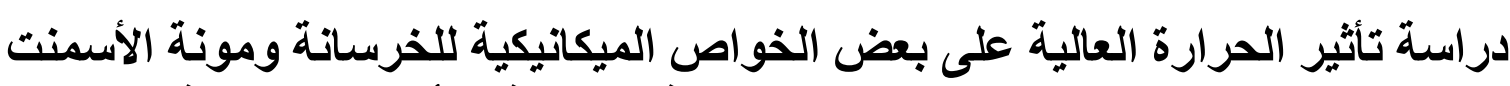

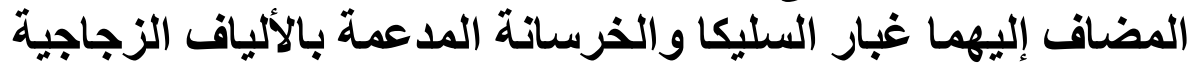

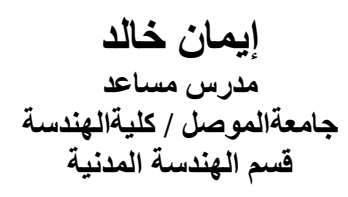

إيمان خالا

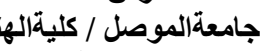

قسم الهندسة المدنية

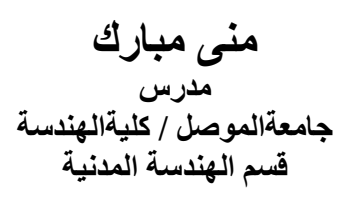

الخلاصة

يشتمل البحث على دراسة عملية لتاثير الحرارة العالية على بعض الخصائص الميكانيكانية والتي تضمنت كل من مقاومة

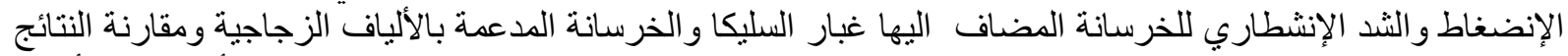

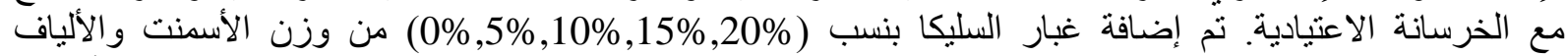

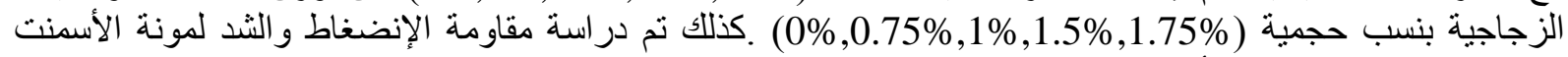

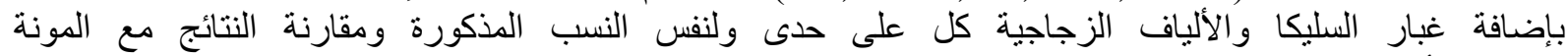

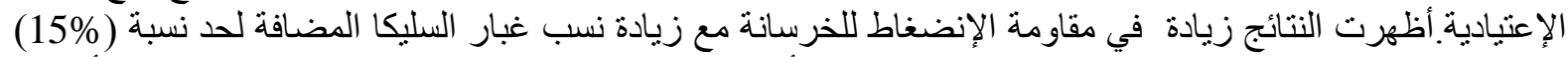

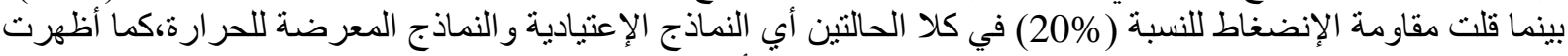

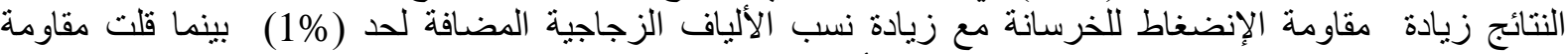
الإنضغاط للنسبتين (1.5\%,1.75\%) في كلا الحالتين أي النماذج الاعتيادية والنماذج المعرضة الإنة للحر ارة.

\section{A Study on the Effect of High Temperature on Some Mechanical Properties of Concrete and Cement Paste with Added Silica Fume and Reinforced with Glass Fiber}

\author{
Samer Sami Majeed \\ Assist. Lecturer \\ Water resources Engineering \\ Department
}

\section{Mona Mobarak \\ Lecturer}

Civil Engineering Department

Mosul Univ. / Engg. College
Eman Khalid

Assist. Lecturer

Civil Engineering

\begin{abstract}
In this research an experimental study was conducted to explore the effect of high temperature on the some mechanical properties such as compressive strength and splitting tensile strength of concrete with added silica fume and reinforced with glass fiber and it as compared with normal concrete. Mixes were prepared by adding silica fume of $(0 \%, 5 \%, 10 \%, 15 \%, 20 \%)$ by weight and glass fiber of $(0 \%, 0.75 \%, 1 \%, 1.5 \%, 1.75 \%)$ by volume . Compressive strength and tensile strength of mortar with the same percentage of silica fume and glass fiber was also studied. The result shown increased concrete compressive strength with increased of silica fume until (15\%) and decreased at (20\%) for both cases with and without heat. Also results shown increased concrete compressive strength with increased of glass fiber until (1\%) and decreased at $(1.5 \%, 1.75 \%)$ for both cases with and without heat.
\end{abstract}

Keywords :Compressive and Tensile strength, Glass fiber, High temperature, Silica fume 


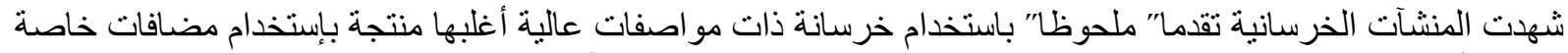

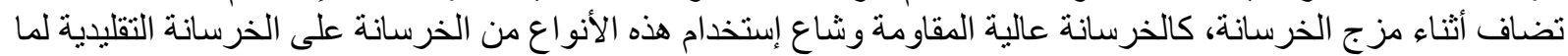

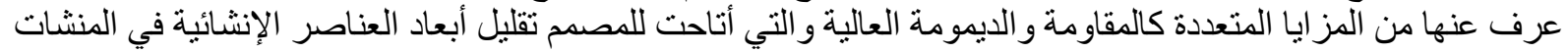

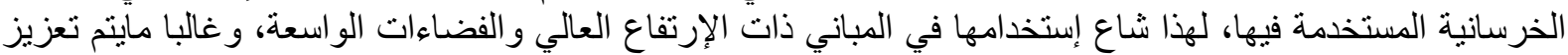

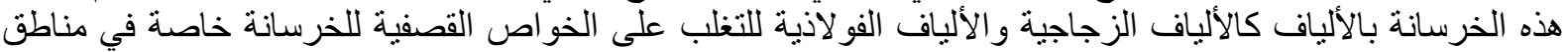

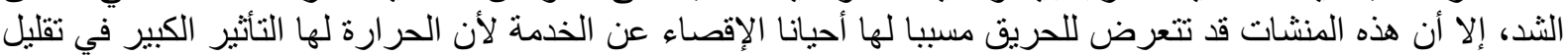

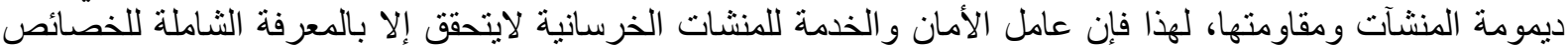

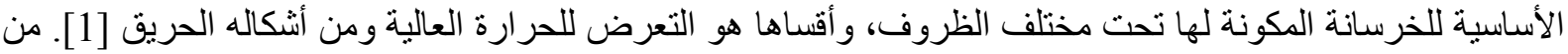

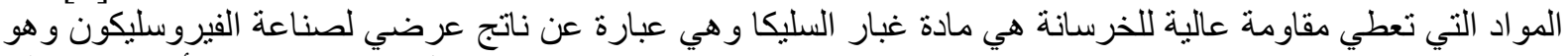

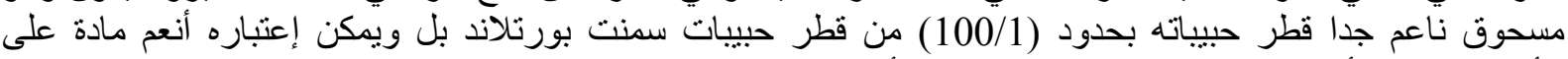

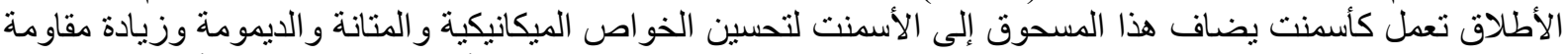

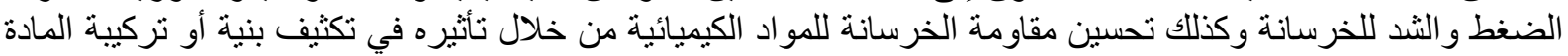

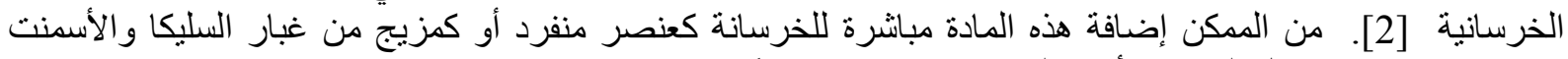

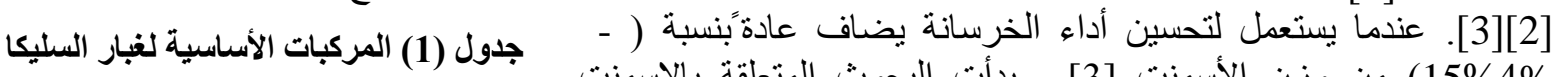

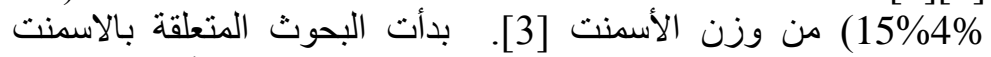

\begin{tabular}{|c|c|}
\hline Oxide & $\%$ \\
\hline SiO2 & 95.3 \\
\hline $\mathrm{Al}_{2} \mathrm{O} 3$ & 0.65 \\
\hline Fe2O3 & 0.28 \\
\hline $\mathrm{CaO}$ & 0.27 \\
\hline $\mathrm{MgO}$ & 0.41 \\
\hline K2O & 0.77 \\
\hline Na2O & 0.26 \\
\hline SO3 & 0.15 \\
\hline
\end{tabular}

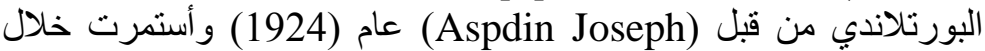

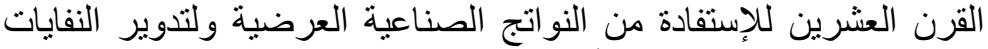

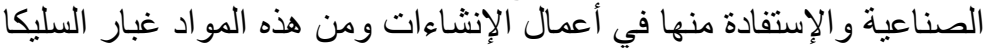

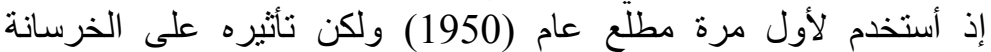

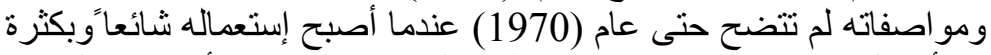

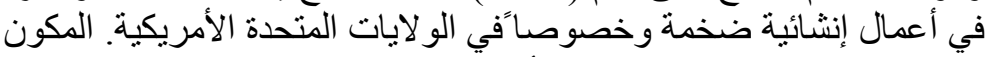

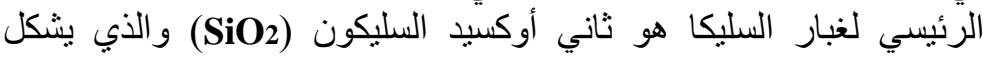

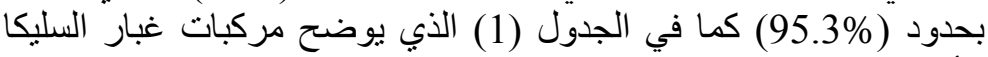

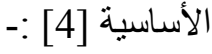

أجريت بحوث ودراسات عديدة حول تأثير إضافة غبار السليكا إلى الخرسانة منها البحث الذي أجراه الباحثان [5hung\&Yunsheng)

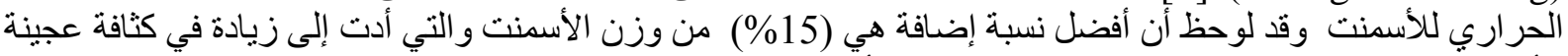

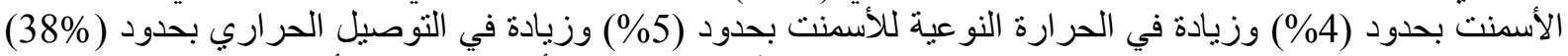

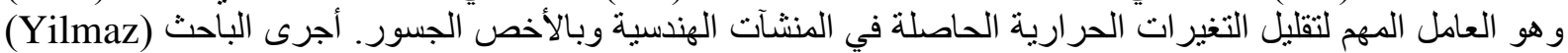

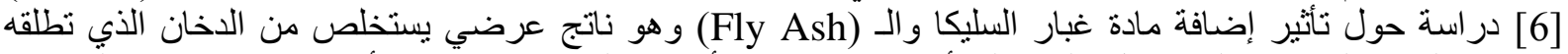

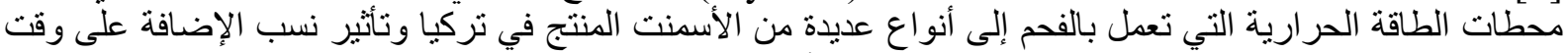

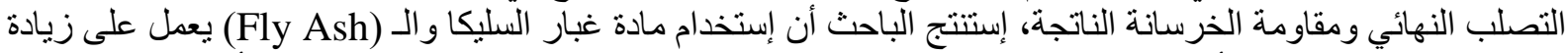

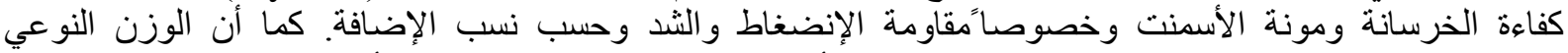

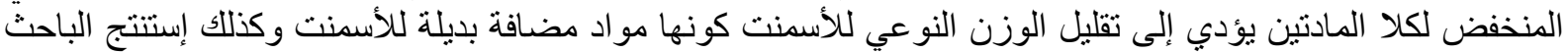

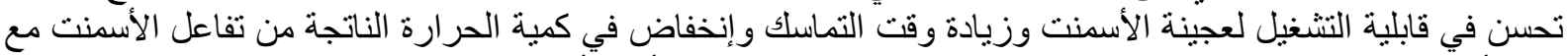

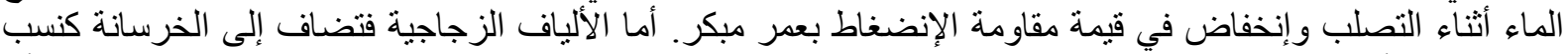

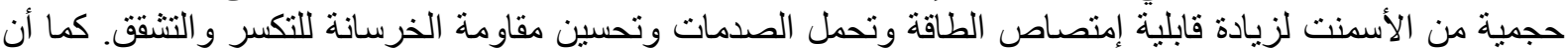

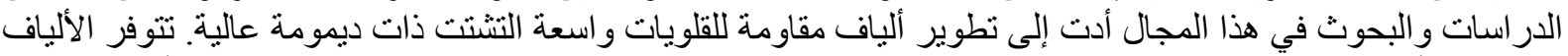

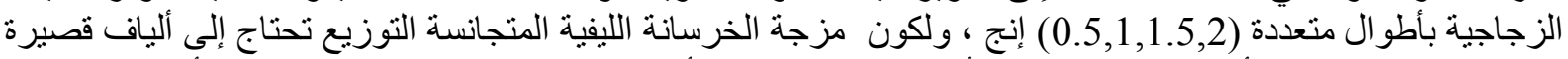

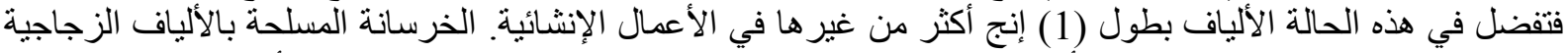

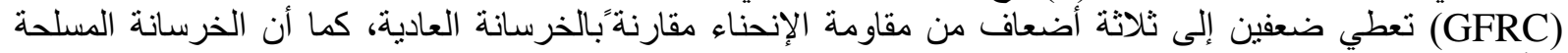

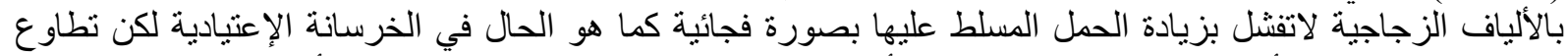

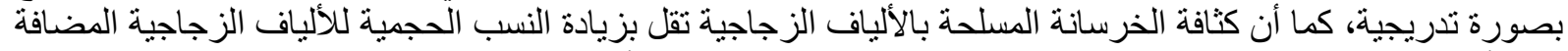

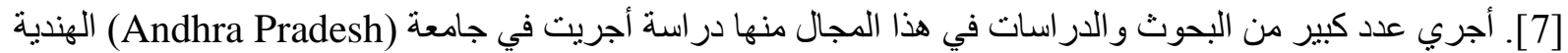

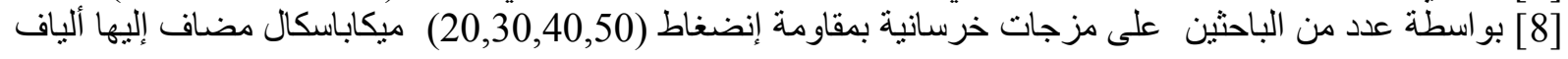


زجاجية بنسبة (0.03\%) فكانت النتائج أن إضافة الألياف الزجاجية يقلل النزيف في الخرسانة الطرية ويقلل إحتمال ظهور

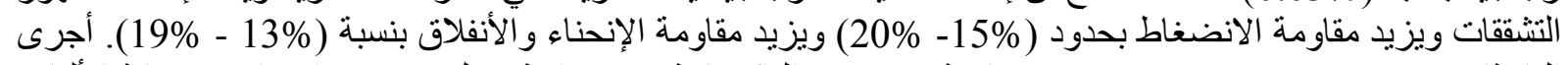

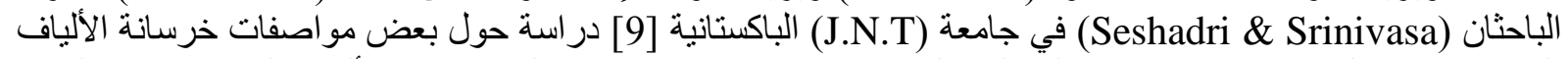

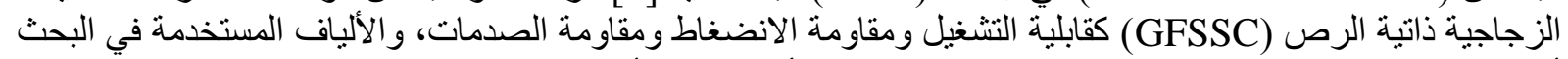

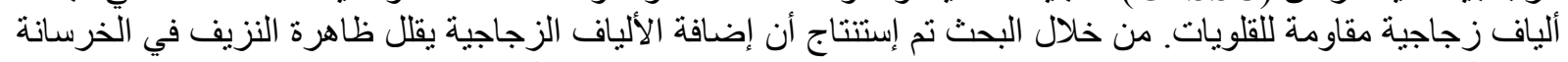

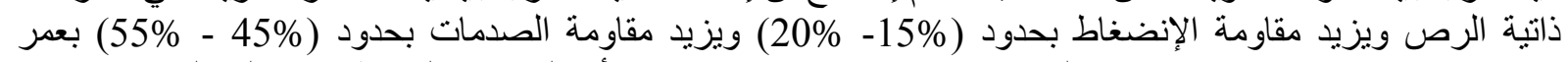

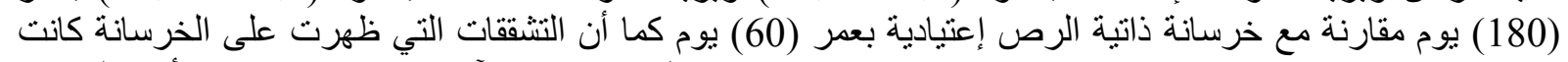

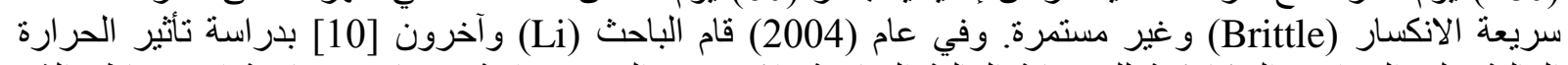

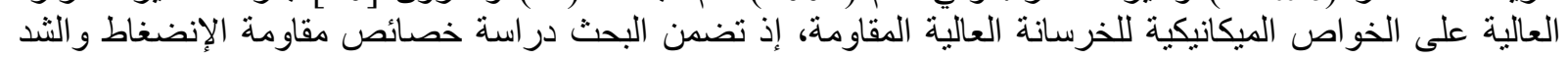

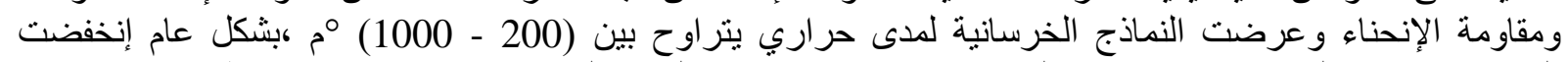

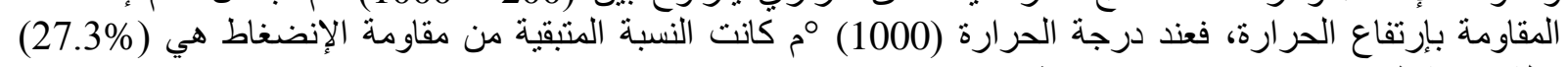
و الثد الإنشطاري بارنفاع الحرارة فيند درجة مقاومة الانحناء (7.4\%).

\section{الهرف من البحث:-}

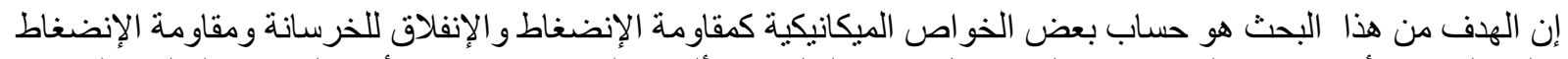

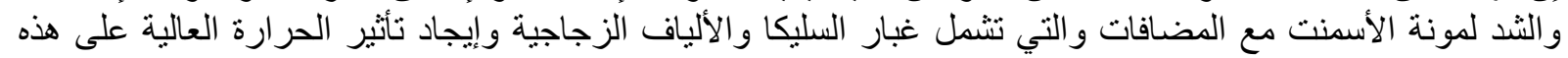
الخواص و إيجاد النسب المثلى لكل من المؤن الد المضافة.

المواد المستخدمة وطريقة الصب :-

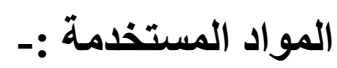

إن المو اد المستخدمة في هذا البحث كانت كما يلي :

1- الأسمنت :- الأسمنت المستخدم أسمنت تركي عادي من نوع ماردين. الجداول (2) و(3) توضح الخواص الفيزيائية و الكيميائية على التو الي لهذا النوع من الأسمنت .

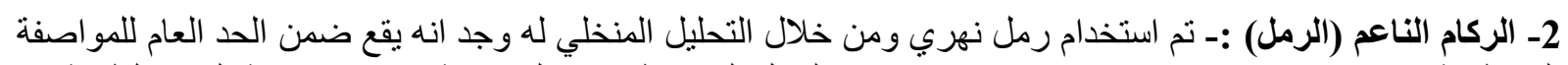

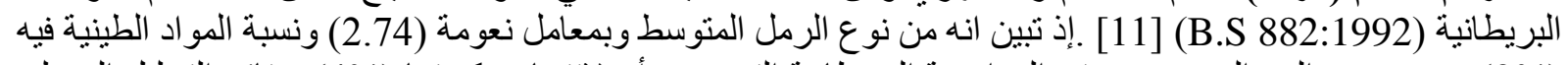

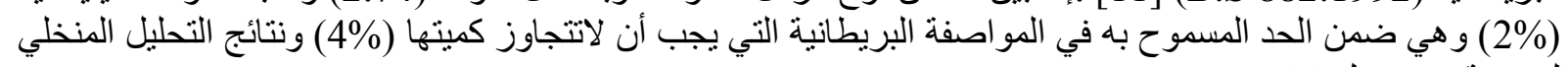

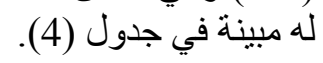

3- الركام الخثن ( الحصى) :- الحصى المستخدم في البحث هو حصى نهري و المسمى محلياً (البحص) ذو شكل المكل مكور

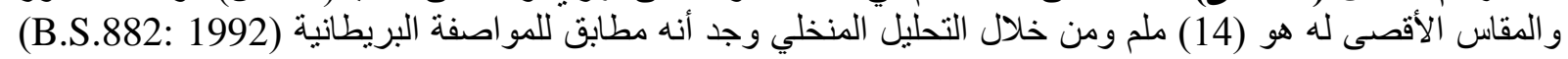

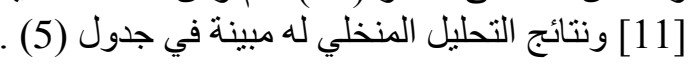
4- الماء :- تم إستخدام ماء الثرب الإعتيادي لمدينة الموصل في الخلطة الخرسانية ومعالجة النماذج. 5- الألياف الزجاجية :- نم إستخدام الألياف الزجاجية بنسب حجية (0.75,1,1.5,1.75) \% على التوالي، والخواص موضحة في جدول (6) :-

6- غبار السليكا :- تم إضافة غبار السليكا بنسب وزنية (0,5,10,15,20)\% على التو الي، والخو اص الفيزيائية و الكيميائية له موضحة في جدول (7) :-

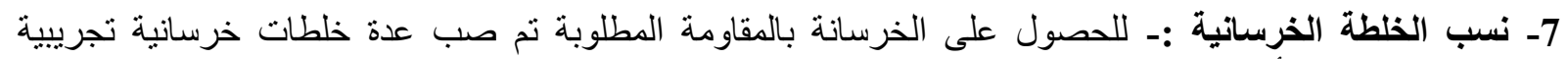

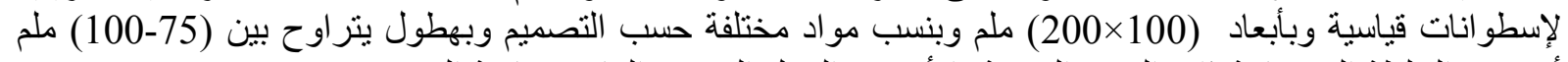

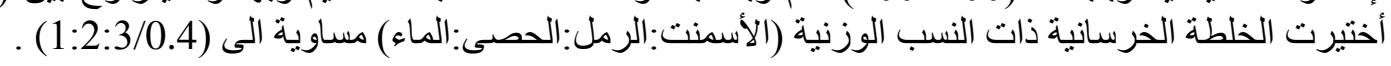


جدول (2) المواصفات الفيزيائية للأسمنت

\begin{tabular}{|c|c|c|}
\hline Test & Result & IQS:5/1984 \\
\hline Consistency & 0.29 & $0.24-0.32$ \\
\hline (min) Initial setting time & 210 & $45 \min (\mathrm{Min})$ \\
\hline (min) Final setting time & 330 & $600 \min (\mathrm{Min})$ \\
\hline (\%) Fineness B.S sieve 170 & 5 & $10 \% \quad(\mathrm{Max})$ \\
\hline \multicolumn{3}{|c|}{ Compressive Strength (MPa) } \\
\hline days 3 & 21 & $15 \mathrm{MPa}$ (Min) \\
\hline days 7 & 28.5 & $23 \mathrm{MPa}(\mathrm{Min})$ \\
\hline
\end{tabular}

جدول (3) المواصفات الكيميائية للأسمنت

\begin{tabular}{|c|c|c|c|c|c|}
\hline $\begin{array}{c}\text { Chemical } \\
\text { Composition }\end{array}$ & \% Value & \% Limits & $\begin{array}{c}\text { Chemical } \\
\text { Composition }\end{array}$ & \% Value & \% Limits \\
\hline $\mathrm{SiO}_{2}$ & 20.30 & ---- & $\mathrm{CaO}$ & 62.00 & ---- \\
\hline $\mathrm{AL}_{2} \mathrm{O}_{3}$ & 3.82 & ---- & $\mathrm{MgO}$ & 2.45 & 6 \\
\hline $\mathrm{Fe}_{2} \mathrm{O}_{3}$ & 4.49 & ---- & $\mathrm{SO}_{3}$ & 0.68 & 2.3 \\
\hline $\mathrm{C}_{3} \mathrm{~S}$ & 64.10 & ---- & $\mathrm{C}_{4} \mathrm{AF}$ & 13.66 & ---- \\
\hline $\mathrm{C}_{2} \mathrm{~S}$ & 9.84 & ---- & $\mathrm{C}_{3} \mathrm{~A}$ & 2.53 & ---- \\
\hline
\end{tabular}

جدول (4) نتائج التحليل المنغلي للرمل

\begin{tabular}{|c|c|c|c|c|c|}
\hline \multirow{2}{*}{$\begin{array}{c}\text { Sieves } \\
(\mathbf{m m})\end{array}$} & \multirow{2}{*}{ \% Passing } & $\begin{array}{c}\text { Specification Limit } \\
\text { (B.S 882-1992) }\end{array}$ & \multicolumn{3}{|c|}{ Additional limits B.S 882-1992 } \\
\cline { 4 - 6 } & & $89-100$ & Coarse & Medium & Fine \\
\hline 5.0 & 100 & $60-100$ & ---- & ----- & ----- \\
\hline 2.36 & 86 & $30-100$ & $30-100$ & $65-100$ & $80-100$ \\
\hline 1.18 & 70 & $15-100$ & $15-54$ & $45-100$ & $70-100$ \\
\hline 0.6 & 49 & $5-70$ & $5-40$ & $5-40$ & $55-100$ \\
\hline 0.3 & 17 & $0-15$ & ----- & ----- & ----- \\
\hline 0.15 & 4 & & & & \\
\hline
\end{tabular}

\begin{tabular}{|c|c|c|}
\hline \multicolumn{3}{|c|}{ جدول (5) } \\
\hline (Sieve size) (mm) & \% Passing & $\begin{array}{c}\text { Specification Limit } \\
\text { (B.S 882-1992) }\end{array}$ \\
\hline$(14 \mathrm{~mm})$ & 97.4 & $90-100$ \\
\hline$(10 \mathrm{~mm})$ & 57.4 & $50-85$ \\
\hline$(5 \mathrm{~mm})$ & 2.1 & $0-10$ \\
\hline$(2.36 \mathrm{~mm})$ & 1 & ---- \\
\hline
\end{tabular}

جدول (6) خواص الألياف الزجاجية

\begin{tabular}{|c|c|c|c|c|}
\hline Cross section & Fiber length & Tensile strength & Young modules & Specific density \\
\hline rectangular & $6 \mathrm{~mm}$ & $400 \mathrm{~N} / \mathrm{mm}^{2}$ & $2600 \mathrm{~N} / \mathrm{mm}^{2}$ & 0.91 \\
\hline
\end{tabular}

جدول (7) الذواص الفيزيائية والكيميائية لفبار السليكا

\begin{tabular}{|c|c|}
\hline Physical properties of silica fume & Chemical properties of silica fume \\
\hline Particle size (typical) $<1$ micron & Amorphous \\
\hline $\begin{array}{c}\text { Bulk density }=130 \text { to } 430 \mathrm{~kg} / \mathrm{m}^{3} \\
\text { Densified = } 480 \text { to } 720 \mathrm{~kg} / \mathrm{m}^{3}\end{array}$ & Silicon dioxide $>85 \%$ \\
\hline Specific gravity $=2.2$ & Trace elements depending upon type of fume \\
\hline Specific surface 15000 to $30000 \mathrm{~m}^{2} / \mathrm{kg}$ & ------------ \\
\hline
\end{tabular}




\section{طريقة صب النماذج الخرسانية :-}

تم إعتماد الخلطة الخرسانية ذات النسب الوزنية (1:2:3/0.4) في عملية الصب ـإن عملية صب النماذج تبدأ بعد تنظيف

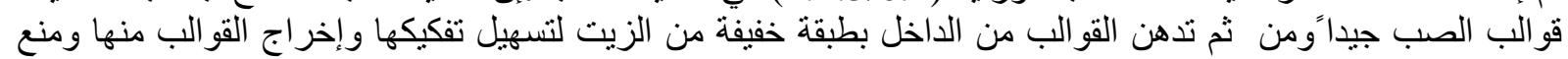

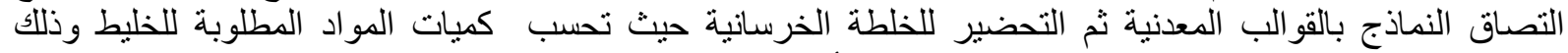

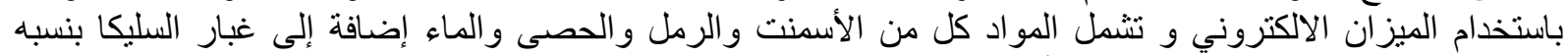

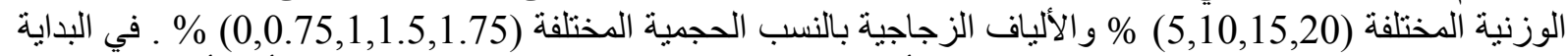

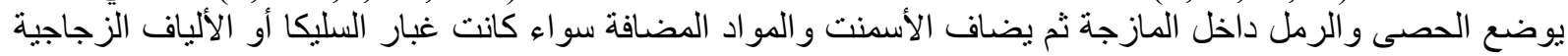

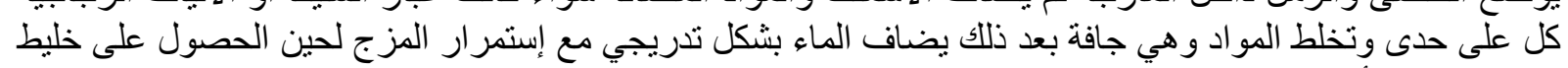

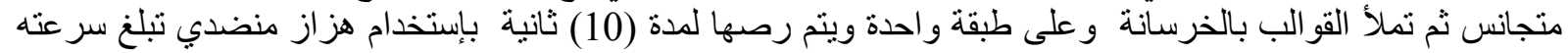

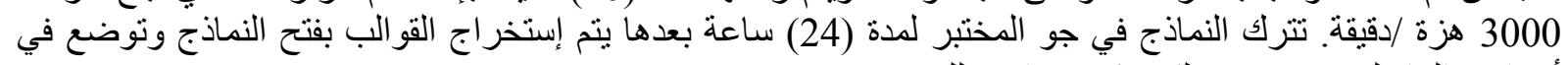

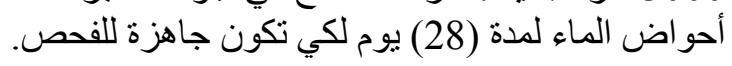

تشخين النماذج الخرساتية الى درجات الحرارة العالية وتبريدها :-

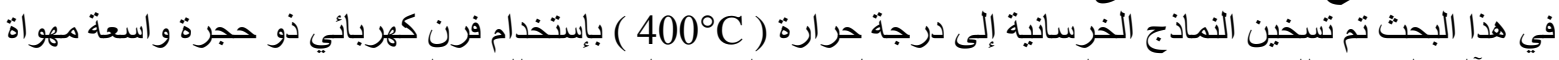

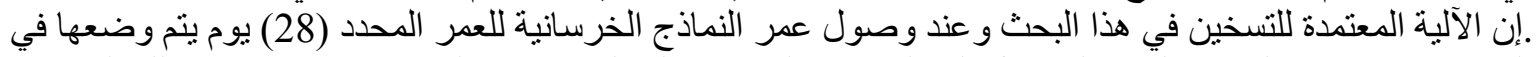

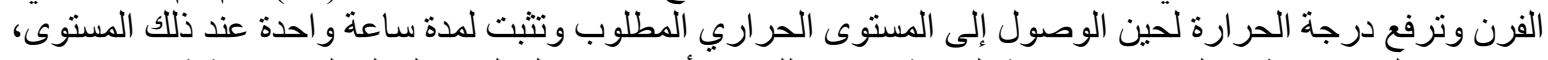

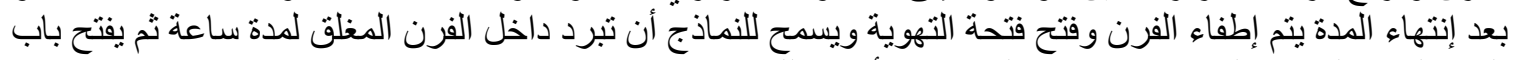

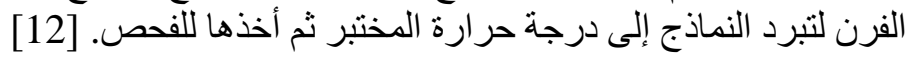

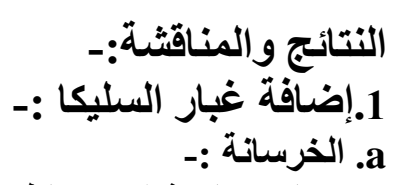

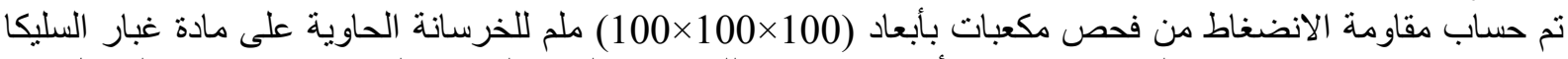
وبنسب (0,5,10,15,20)\% لنماذج اعتيادية وأخرى معرضة للحر ارة ولنفس النسب و النتائج موضحة في الجدول

و الثكل (1) (1) (1) (1)

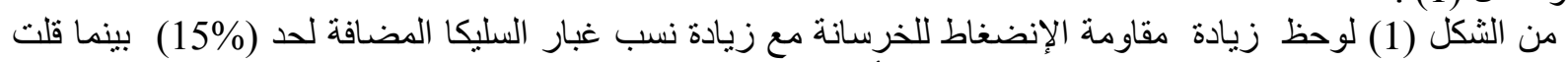

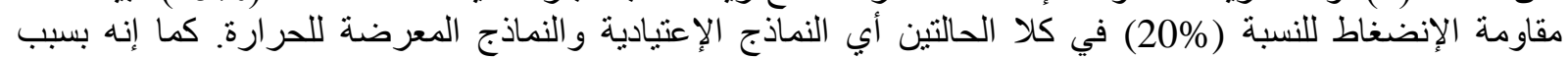

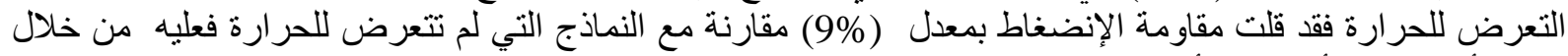

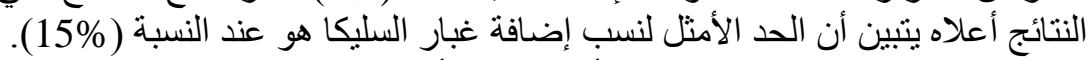

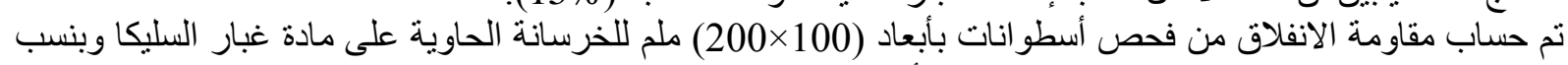

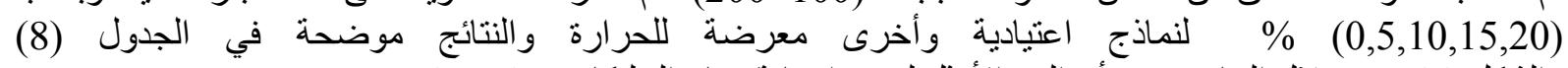
والثكل (2) .من خلال النتائج يتبين أن الحد الأمثل لنسب إضافة غبار السليكا عند (15\%).
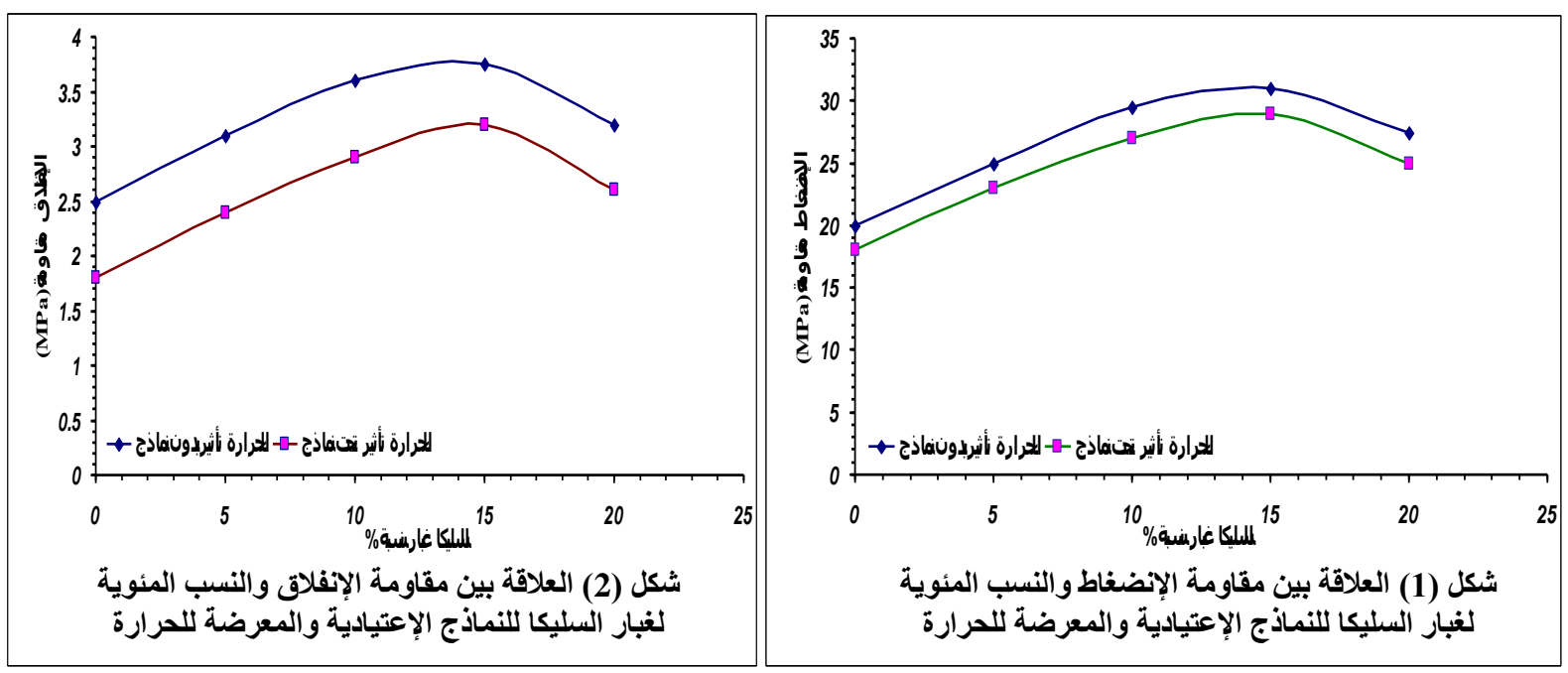
من خلال النتائج لوحظ أن مقاومة الإنفلاق هي بمعدل (8.5) \% من من مقاومة الإنضغاط بالنسبة للنماذج الإعتبادية وبمعدل (10) \% للنماذج المعرضة للحرارة وكما مبين في الثكلين (3) و (4) هن (4).
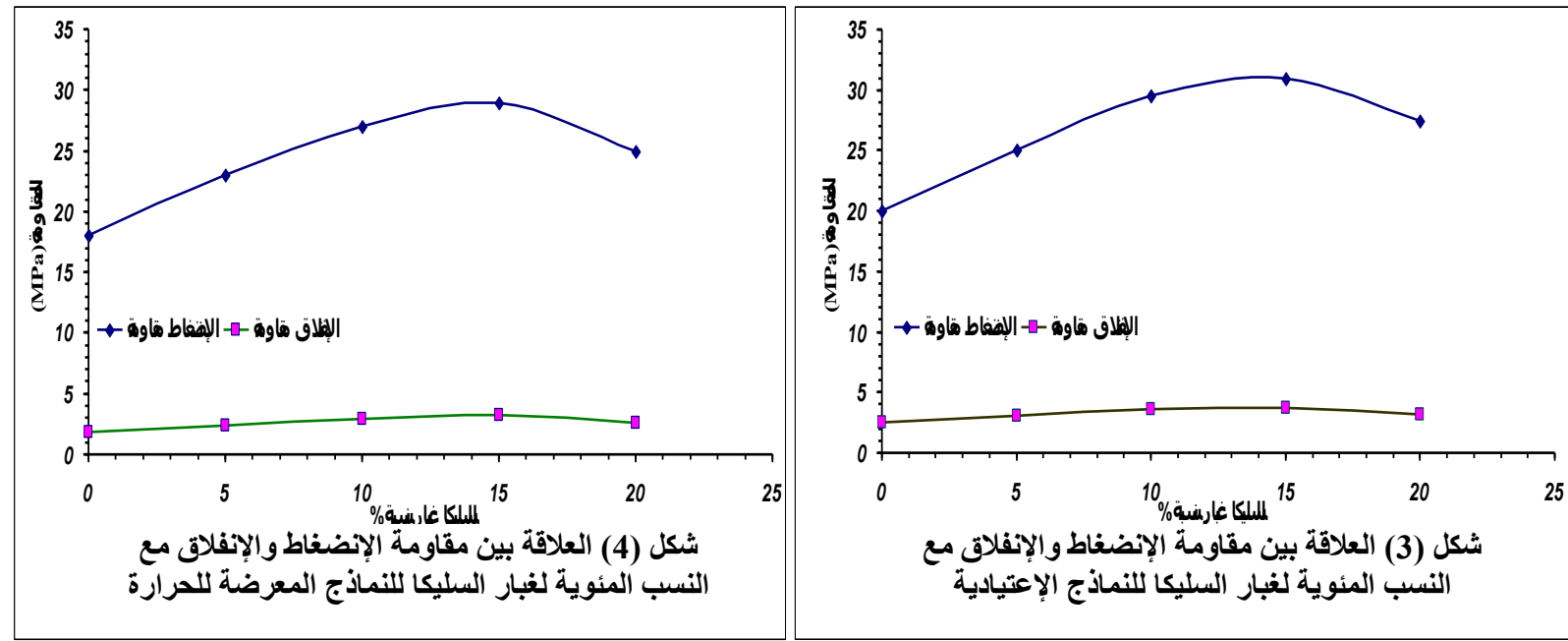

جدول (8) مقاومة الإضغاط والإنفلاق للخرسانة الحاوية على غبار السليكا مع ويدون تأثير الحرارة

\begin{tabular}{|c|c|c|c|c|}
\hline \multicolumn{3}{|c|}{ Normal concrete } & \multicolumn{2}{c|}{ Concrete subjected to heat } \\
\hline Silica fume \% & fc $^{\prime}($ MPa) & ft (MPa) & fc' $^{\prime}$ (MPa) & ft (MPa) \\
\hline 0 & 20 & 2.5 & 18 & 1.8 \\
\hline 5 & 25 & 3.1 & 23 & 2.4 \\
\hline 10 & 29.5 & 3.6 & 27 & 2.9 \\
\hline 15 & 31 & 3.75 & 29 & 3.2 \\
\hline 20 & 27.4 & 3.2 & 25 & 2.6 \\
\hline
\end{tabular}

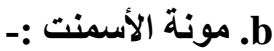

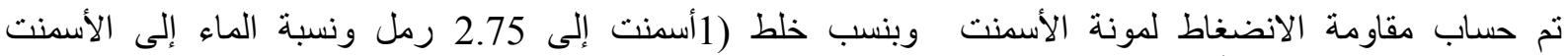

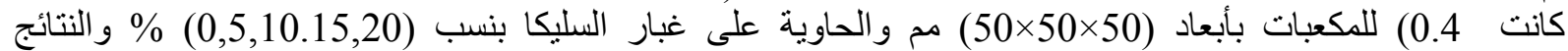

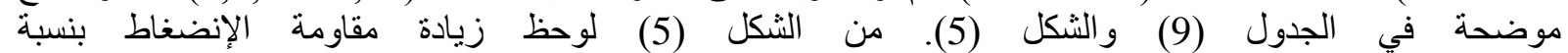
(60,112,134,78) \% النسب غبار السليكا المضافة وقد بينت النتائج أن النسبة المثلى لغبار السليكا إلى الأسمنت بين

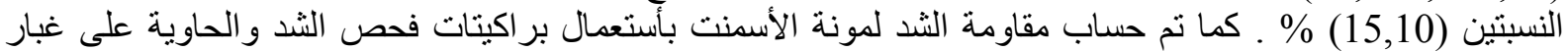

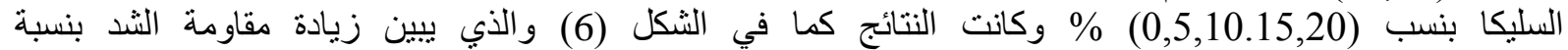
(54,92,112,73)

جدول (9) مقاومة الإنضغاطوالثد لمونة الأسمنت الحاوية على غبار السليكا

\begin{tabular}{|c|c|c|}
\hline \multicolumn{3}{|c|}{ Mortar } \\
\hline Silica fume\% & fc' $^{\prime}(\mathbf{M P a})$ & $\mathbf{f t}$ (MPa) \\
\hline $\mathbf{0}$ & 24.4 & 2.6 \\
\hline 5 & 39 & 4 \\
\hline 10 & 51.73 & 5 \\
\hline 15 & 57 & 5.5 \\
\hline 20 & 43.4 & 4.5 \\
\hline
\end{tabular}



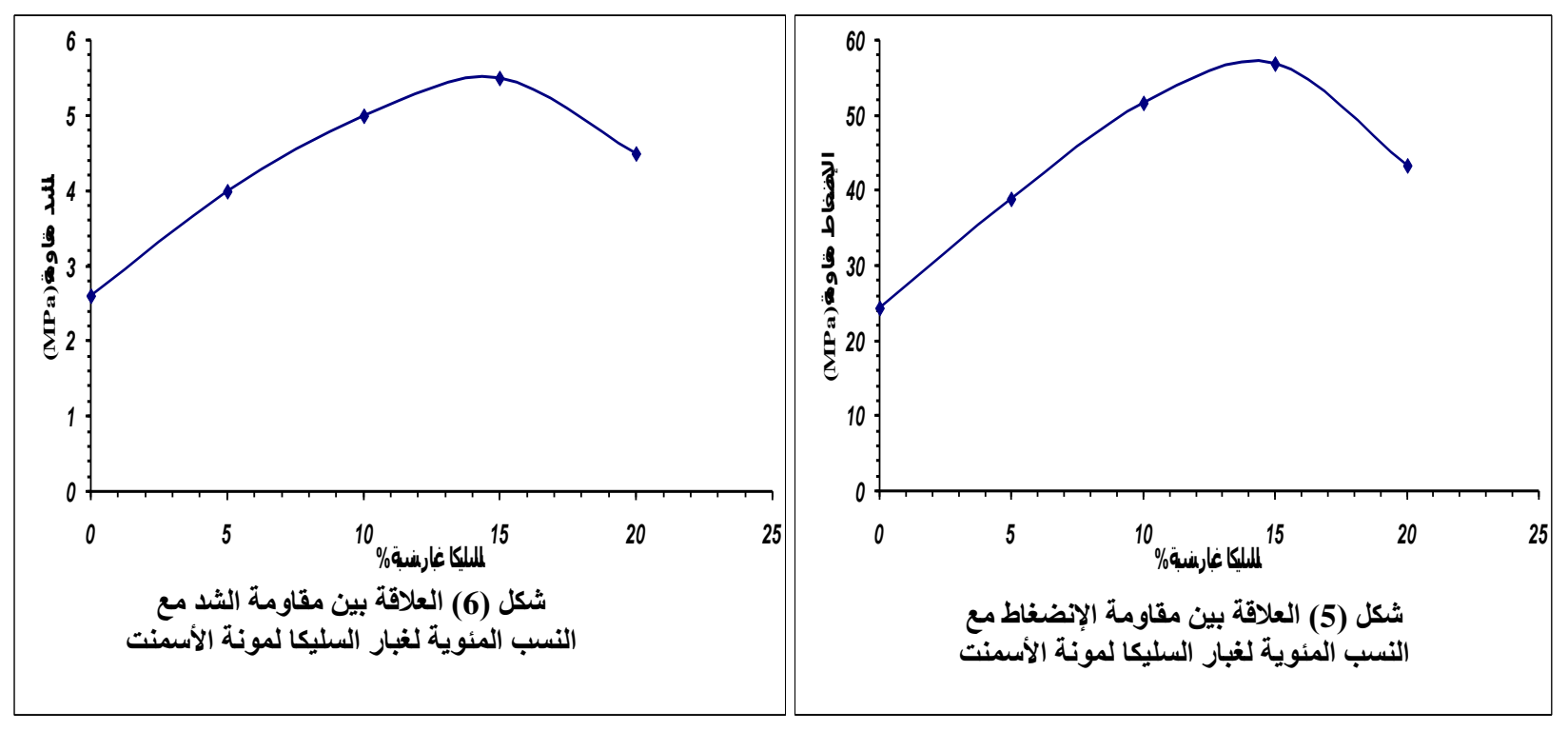

\section{2.إضافة الألياف الزجاجية:-

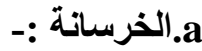

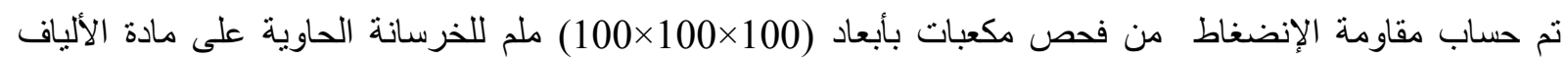

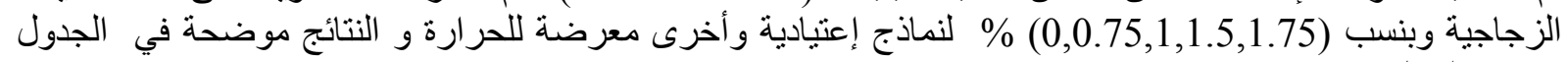

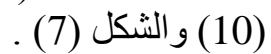

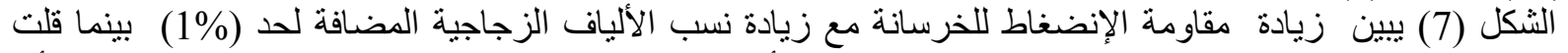

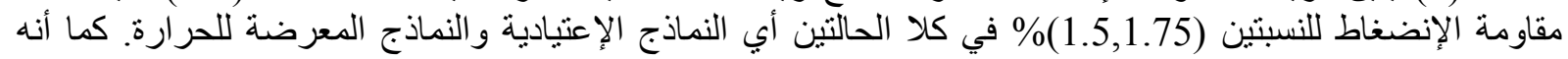

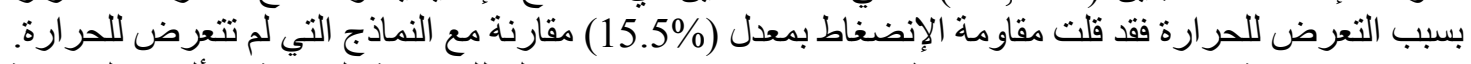

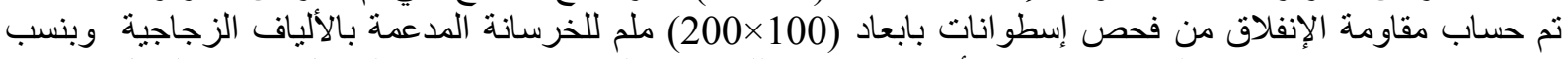

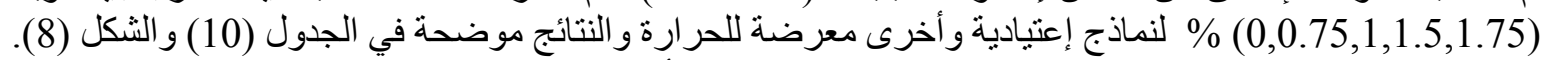

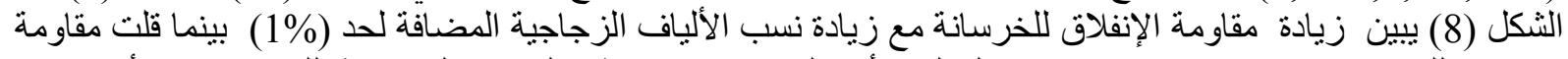

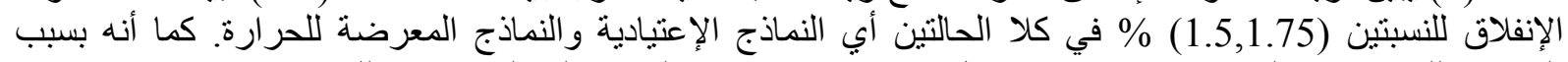

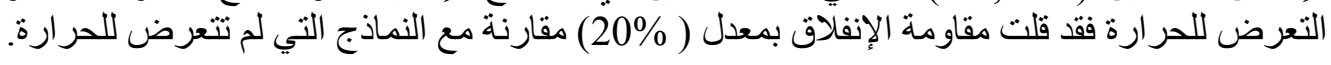

جدول (10) مقاومة الإنضغاط والإنفلاق للخرسانة الحاوية على الألياف الزجاجية مع وبدون تأثير الحرارة

\begin{tabular}{|c|c|c|c|c|}
\hline \multicolumn{3}{|c|}{ Normal concrete } & \multicolumn{2}{c|}{ Heated concrete } \\
\hline Glass fiber \% & fc' $^{\prime}$ (MPa) & ft (MPa) & fc $^{\prime}$ (MPa) & ft (MPa) \\
\hline 0.00 & 30 & 2.62 & 25.3 & 2.2 \\
\hline 0.75 & 33.8 & 3.42 & 29.47 & 2.69 \\
\hline 1.00 & 34.8 & 3.7 & 30.5 & 2.81 \\
\hline 1.50 & 34.35 & 3.5 & 29.56 & 2.8 \\
\hline 1.75 & 31 & 2.5 & 24.18 & 2.18 \\
\hline
\end{tabular}






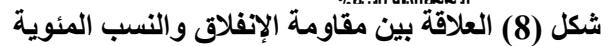

للألياف الزجاجية للنماذج الإعتيادية والمعرضة والمرة للحرارة

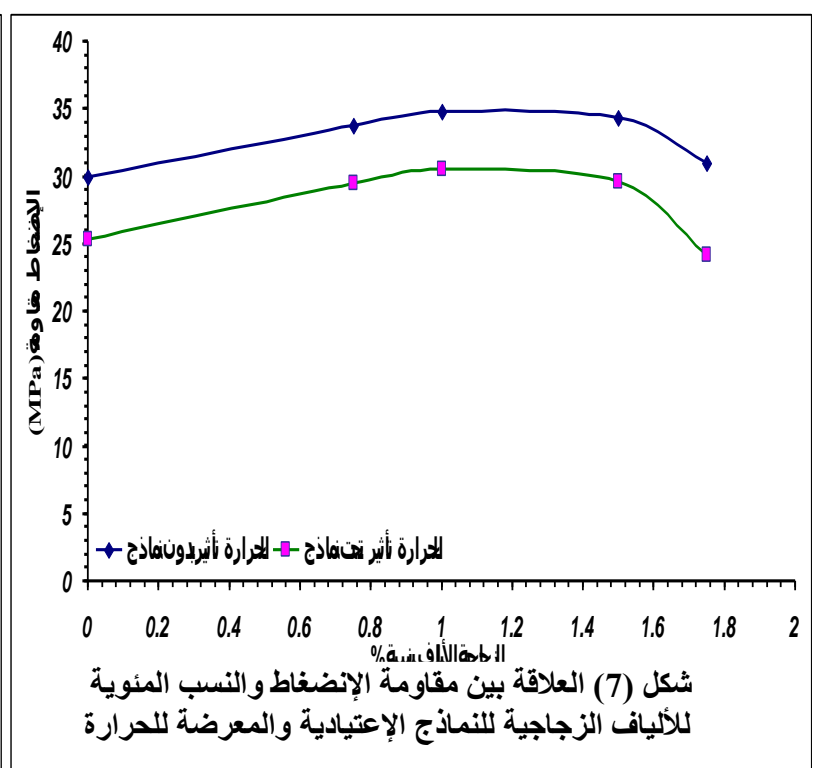

من خلال النتائج لوحظ ان مقاو مة الثد هي بمعدل (9.5) \% من مقاد مقاومة الانضغاط بالنسبة للنماذج الإعنيادية وبمعدل (11)

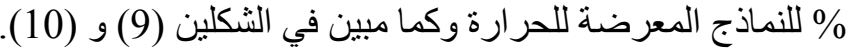
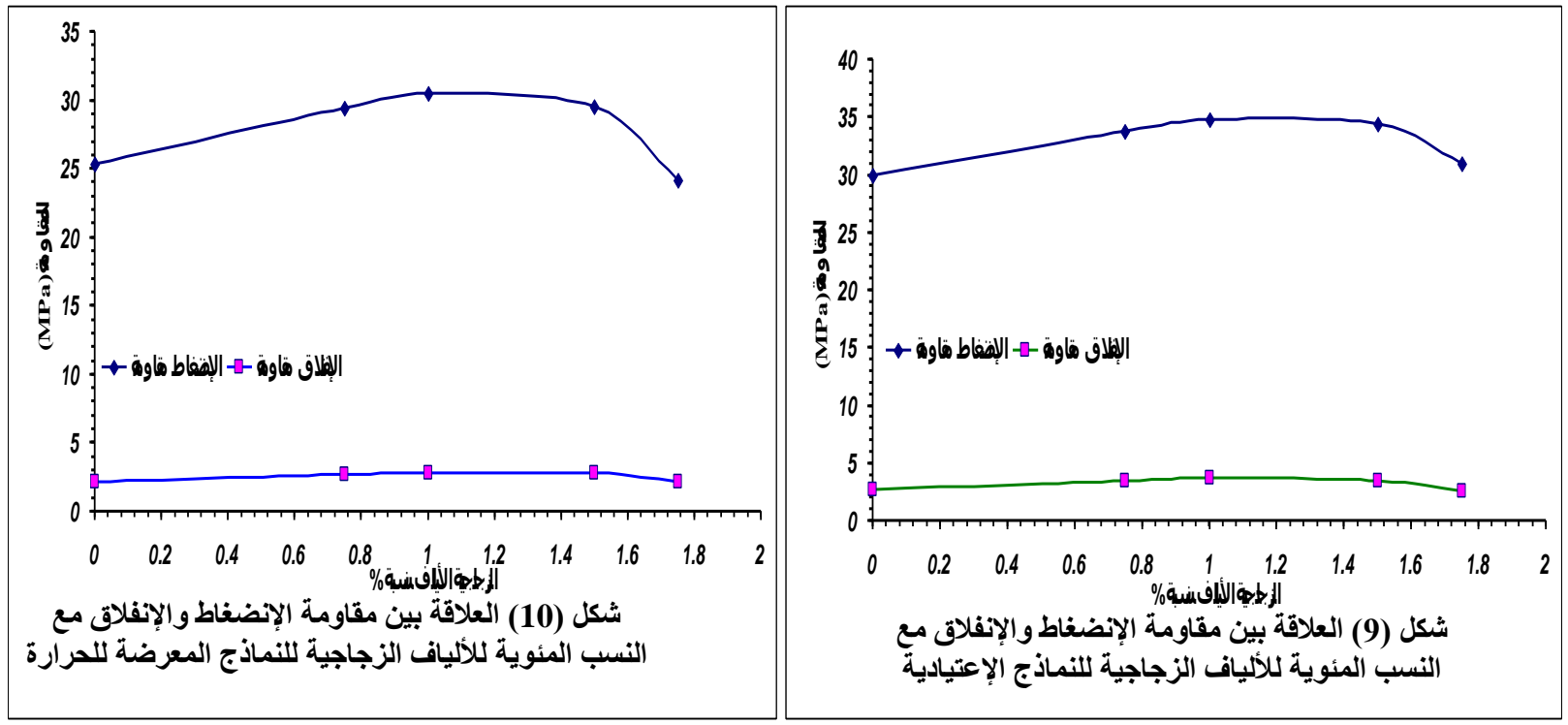

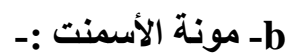

كما تم حساب مقاومة الإنضغاط لمونة الأسمنت وبنسب خلط (1 أسمنت إلى 2.75 رمل ونسبة الماء إلى الأسمنت كانت

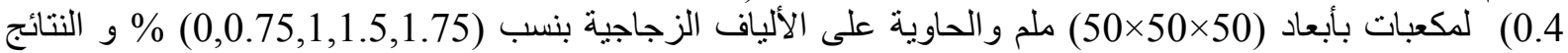

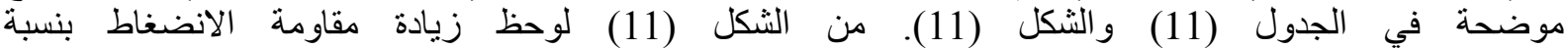
35,37,23، 8) \% لنسب الألياف الزجاجية المضافة وقد بينت النتائج أن النسبة المثلى للألياف الزجاجية هي (1\%).

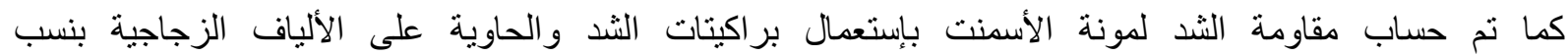

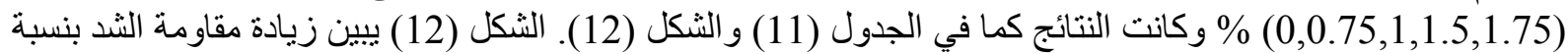
(34,41,39,17 \% لنسب الألياف المضافة وقد بينت النتائج أن النسبة المتلى للألياف الزجاجية المضافة هي (1\%). 
جدول (11) مقاومة الإنضغاط والثد لمونة الأسمنت الحاوية على الألياف الزجاجية

\begin{tabular}{|c|c|c|}
\hline \multicolumn{3}{|c|}{ Mortar } \\
\hline Glass fiber\% & fc $^{\prime}($ MPa $)$ & ft (MPa) \\
\hline 0.00 & 24.46 & $\mathbf{2 . 3 9}$ \\
\hline 0.75 & 33.06 & 3.20 \\
\hline 1.00 & 33.92 & 3.37 \\
\hline 1.50 & 30.20 & 3.20 \\
\hline 1.75 & 26.25 & 2.80 \\
\hline
\end{tabular}

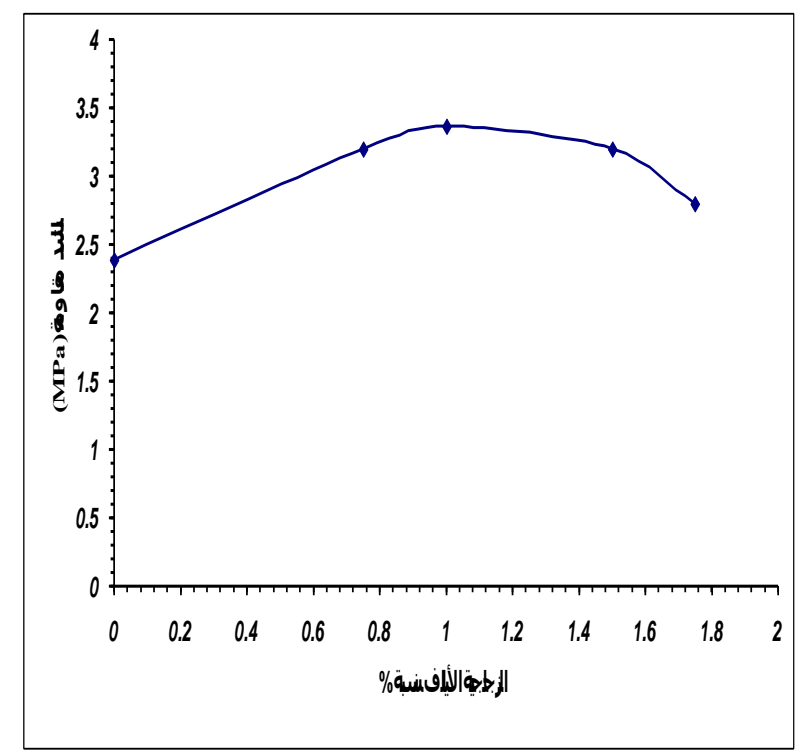

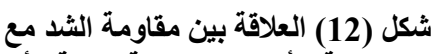

النسب المئوية للألياف الزجاجية لمونة بلة الأسمنة

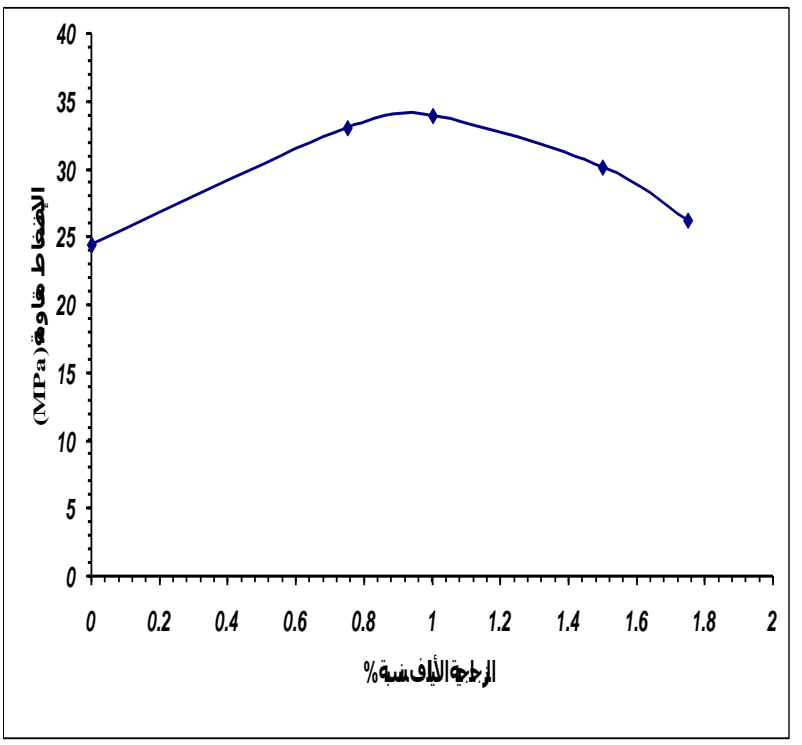

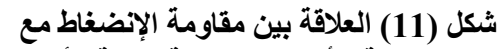
النسب المئوية للألياف الزجاجية لمقاومية الإنة الأسمنت

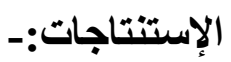

من خلال النتائج التي تم الحصول عليها يمكن إستنتاج مايلي :-

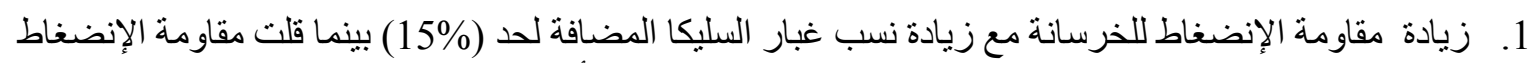

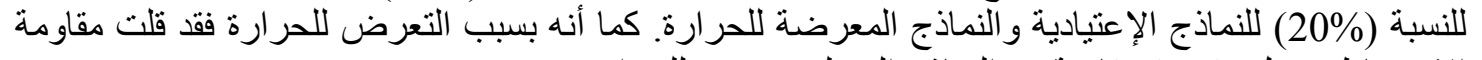



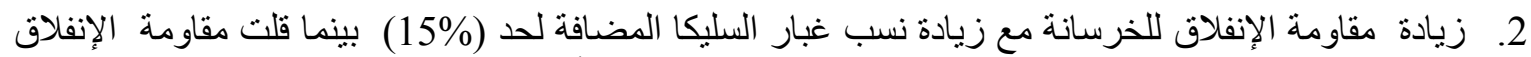

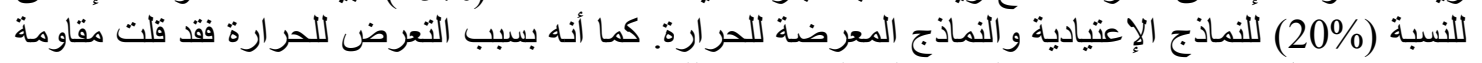

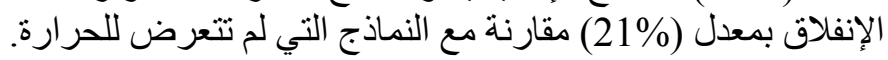

3. إن مقاومة الإنفلاق للخرسانة المضاف إليها غبار السليكا هي بمعدل (8.5\%) من مقاومة الإنضغاط بالنسبة للنماذج الخرسانية الإعتيادية وبمعدل (10\%) للنماذج الخرسانة الخدانية المعرضة للحر ارة.

4. بالنسبة لمونة الأسمنت فقد زادت مقاومة الانضغاط بنسبة ( 60,112,134,78) \% \% لنسب السليكا المضافة كما بينت النتائج ان النسبة المنلى لغبار السليكا المضافة هي بين (10,15) \% 


$\begin{array}{llll}\text { Al-Rafidain Engineering } & \text { Vol.21 } & \text { No. } 4 & \text { August } 2013\end{array}$

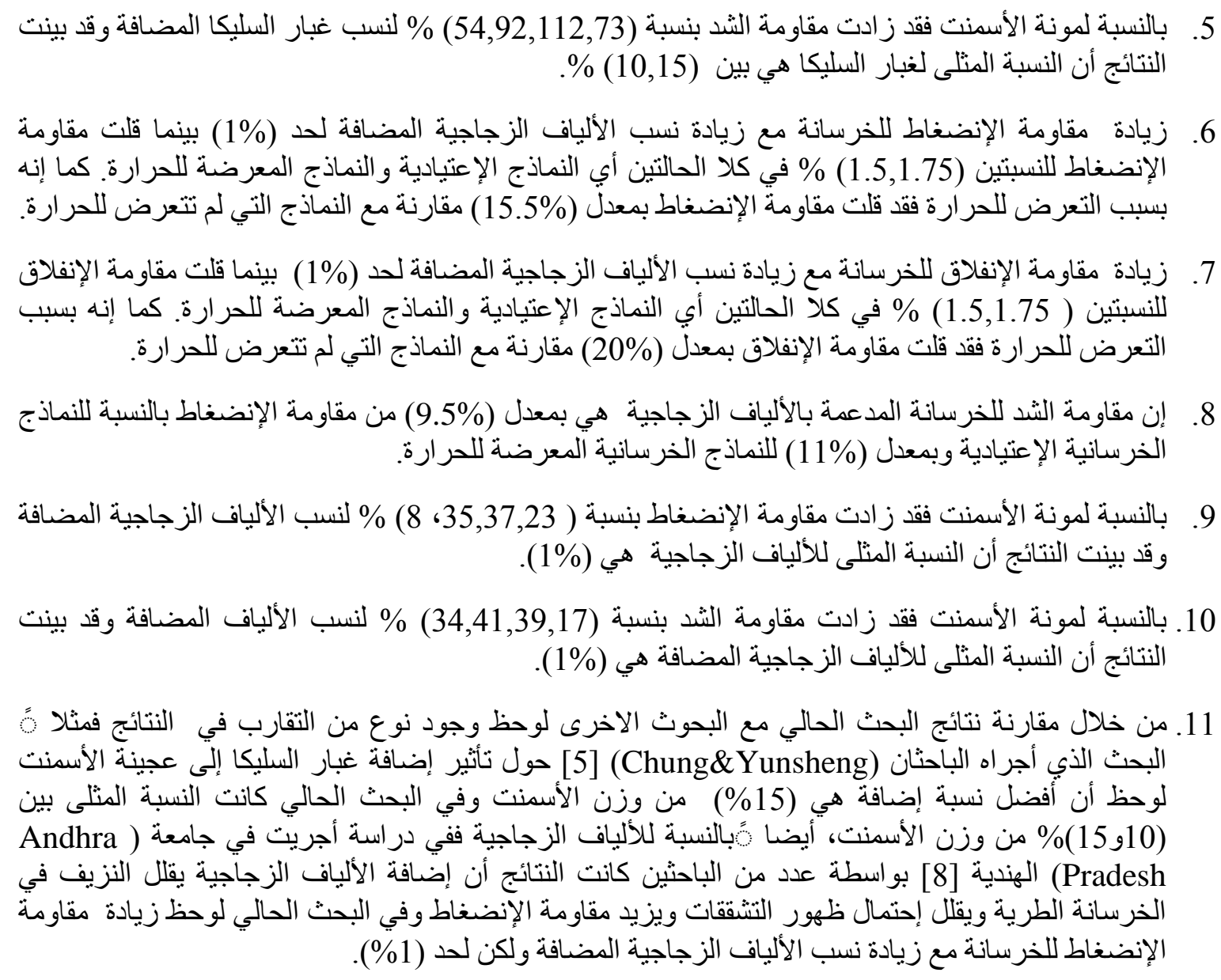

1. Abdul Rahman,R.B. ,'Effect of Elevated Temperature on Some Properties of Air Entrained Steel-Fibers Reinforced Concrete”, Thesis, University of Mosul ,2007.

2. ACI - Committee234, " Guide for the Use of Silica Fume in Concrete ", (ACI 234R-06) American Concrete Institute, April, 2006, pp.1-3.

3. Arnon Chaipanich, “ Silica Fume: Its Role in Cement and Concrete Technology " , Department of Physics, Chiang Mai University, Thailand.

4. Malhotra, V. M. and P. K. Mehta 1996. Pozzolanic and cementitious materials. Advances in Concrete Technology, Gordon and Breach Publishers, Vol 1.

5. Yunsheng $\mathrm{Xu}$ and Chung, "Cement of high specific heat and thermal conductivity, obtained by using silane and silica fume as admixtures ", Department of Mechanical and Aerospace Engineering, State University of New York at Buffalo, April 2000, PP 11751178.

6. Yilmaz Kocak, “A study on the effecy of fly ash and silica fume substituted cement paste and mortar ", Department of Construction Education , Faculty of Technical Education, Duzce University, April 2010, pp. 990-998. 
7. Wallace Neal, " Glass fiber reinforced concrete (GFRC), A new composite for construction ", Construction Specification Institute, Minneapolis University.

8. Chandramouli, Srinivasa Rao, Pannirselvam, Seshadri Sekhar, Sravana, "Study on Strength and Durability Characteristics of Glass Fiber concrete ", International Journal of Mechanics and Solids, ISSN 0973-1881 Volume 5, Number 1 (2010), pp.15-26.

9. Srinivasa Rao, Seshadri Sekhar, "Impact Strength and Workability Behaviour of Glass Fiber Self Compacting Concrete ", International Journal of Mechanics and Solids, ISSN 0973-1881 Volume 3, Number 1 (2008), pp.61-74.

10. Li,M., Qian, C and Sun, W., "Mechanical Properties of high strength concrete after fire “, Cement and Concrete Research,Vol. 34, 2004, pp.1001-1005.

11. British Standards Institute, B.S 882-1992 “ Aggregates from Natural Sources for Concrete ".

12. Mohamedbhai ,G.T.G., " Effecte of Exposure time and Rates of Heating and Cooling on Residual Strength of Heated Concrete ", Magazine of Concrete research,Vol. 8, No. 136, September 1986, pp.151-158.

تم اجراء البحث في كلية الهندسة = جامعة ألموصل 